\title{
Geodesic ray-tracing in white matter fiber crossing region using decomposition of 4 th order tensor
}

\author{
Avinash Bansal ${ }^{\mathrm{a}}$, Sumit Kaushik ${ }^{\mathrm{b}}$, Temesgen Bihonegn ${ }^{\mathrm{a}}$, and Jan Slovák ${ }^{\mathrm{a}}$ \\ ${ }^{a}$ Masaryk University, Faculty of Science, Departement of Mathematics and Statistics, Brno, \\ Czech Republic \\ ${ }^{\mathrm{b}}$ Czech Technical University in Prague, Faculty of Electrical Engineering, Czech Republic
}

\begin{abstract}
The streamline methods in Diffusion tensor imaging (DTI) use principal eigenvectors for tracking white matter fibers. In comparison, the geodesics in a multivalued ray-tracing method are closer to the actual underlying white matter fibers. This method provides multiple solutions in the form of geodesics in a Riemannian space. These geodesics are robust in tracking high curvature structures in the presence of noise. In the DTI modality, the 2nd order tensor fails to model the heterogeneous regions, e.g., crossing/merging fibers. Therefore, the ray-tracing method is limited to guide the geodesics in these regions. This work, as a first step, utilizes 4 th order tensor approximation for the white matter regions. Subsequently, a non-linear optimization is performed to decompose 4th order tensors into multiple 2nd order tensors keeping their symmetric positive definite property. We are using the initial fiber directions from the diagonal components of the 4th order tensor and use the 4th order tensor decomposition for fiber tracking. The experimental results on synthetic images show that geodesics can traverse in heterogeneous and high curvature structures.
\end{abstract}

Keywords: DW-MRI, DTI, tensor decomposition, 4th order tensor, fiber tracking, geodesics, Riemannian space

\section{INTRODUCTION}

Diffusion-weighted magnetic resonance imaging (DW-MRI) is a non-invasive tool in studying microstructures in the human brain. There exist many acquisition techniques and models based on DW-MRI. In our current work, we are interested in the high angular resolution (HARDI) technique ${ }^{1}$. The higher order tensors (HOTs) are considered for modelling water diffusion in multi-fiber regions. These HOTs should be symmetric positive definite since the diffusion profile represent a physical process. These tensors can describe water diffusion in complex micro-structures better than Gaussian based DTI model. Örzaslan et $\mathrm{al}^{2}$ observed that the coefficients of a lower order tensor can be computed linearly from those of higher order. It allows a significant reduction in acquisitions. Another useful feature of HOTs is that their coefficients can be represented in terms of spherical harmonics.

Most of the tracking methods in DTI modality ${ }^{3}$ are based on principal eigenvector directions of 2nd order tensors. These methods are not efficient in high curvatures regions and perform poor in presence of noise. In case where tensors are slightly anisotropic, they causes deviation from the actual water diffusion path. Another approach, known as geodesic ray-tracing is based upon finding geodesics with respect to a suitably chosen metric. The ray-tracing method was introduced in the works by Sepasian et $\mathrm{al}^{4,5}$. The geodesics are eventually used for reconstruction of fibers. This method computes multiple geodesics between two points in the anisotropic region, they are robust to noise and traverse closer to the actual path in curved white matter structures. It relies on solving Euler-Lagrange (EL) equations which accounts for the local changes in path.

A geodesic is a path locally minimizing length between two points. A metric tensor needs to be chosen on Riemmanian space to solve the geodesic equation. Faster diffusion of water molecules in a direction locally represents a shorter path that can be considered a geodesic. In earlier studies ${ }^{6,7}$, such metric tensor is defined

Corresponding author: kaushsum@fel.cvut.cz 
as the inverse of the 2nd order diffusion tensor. This choice of metric has limitations in anisotropic regions with high curvatures requiring the sharpening of diffusion tensors ${ }^{8}$.

In this work, we utilize the observation from our previous work ${ }^{9,10}$ which suggest that the principal directions of the diagonal components (DC), obtained by flattening a 4th order tensor, can be used to extract main fiber directions. As a contribution, we modified the ray-tracing method to perform tracking in crossing regions. The 4th order tensors used for modelling crossing fiber bundles are decomposed into 2nd order tensors using nonlinear optimization technique ${ }^{11}$. This method originally relied on fixing the principal eigenvector directions from the maxima of the orientation distribution function. We suggest to obtain these principal directions from the DC method. The maximum eigenvector of the components serves as the initial input required in the decomposition process. The experiments on various simulated images indicate that the resulting tensors can be used to guide the geodesics in crossing fibers at small angles and those with high curvature.

\section{RELATED WORK}

\subsection{Geodesic equation}

By definition, a geodesic is a curve $x(t)$ between two points in a geometric space, which minimizes the length of path. In the Riemannian manifold defined by a metric tensor $g$, this path can be evaluated by solving 2 nd order ODE equations.

$$
\ddot{x}^{i}+\Gamma_{k l}^{i} \dot{x}^{k} \dot{x}^{l}=0,
$$

where

$$
\dot{x}^{i}=\mathrm{d} x^{i} / \mathrm{d} t
$$

is velocity and $\Gamma_{i j}^{k}$ are the Christoffel symbols. It is defined by

$$
\Gamma_{i j}^{k}=\frac{1}{2} \sum_{\sigma=1}^{3} g^{k l}\left(\frac{\partial g_{j l}}{\partial x^{i}}+\frac{\partial g_{i l}}{\partial x^{j}}-\frac{\partial g_{i j}}{\partial x^{l}}\right)
$$

where $g_{i j}$ denotes the entries of the Riemannian metric, $g$ and $g^{i j}$ denotes that of the inverse metric, $g^{-1}$.

In our experiments, we restrict to $2 \mathrm{D}$ fibers which need 12 Christoffel symbols, six for each direction due to indices symmetry $\left(\Gamma_{i j}^{k}=\Gamma_{j i}^{k}\right)$. The above second order differential equation can be written as a set of first order equations which are finally solved using Runge-Kutta numerical method. Once initial position and direction are supplied to the ODE solver, it evaluates the next position and direction locally. For keeping the initial shooting direction precise, geodesics are restricted to a cone. As the curve progresses it tends to deviate from the actual path due to cumulative errors. For this reason, we chose to rescale the metric tensor using sigmoid function under scalar Hilbert anisotropy $(\mathrm{HA})^{9}$.

\subsection{Diffusion model}

The Stejskal-Tanner equation ${ }^{12}$, modeling water diffusion in tissue micro structures, is a mono-exponential model given by

$$
S=S_{0} \exp (-b d(\mathbf{g}))
$$

where, $S$ is attenuated signal when gradient pulse is applied, $d(\mathbf{g})$ the apparent diffusion coefficient (ADC) and $b$ is an acquisition constant.

The diffusivity function of 4 th order tensors can be expressed using the standard notation of quartic forms. When modeling the diffusion process with HOT, the diffusivity function can be written as:

$$
d(\mathbf{g})=\sum_{i+j+k=4} \mathbf{T}_{i, j, k} g_{1}^{i} g_{2}^{j} g_{3}^{k}
$$

where $\mathbf{g}=\left[g_{1}, g_{2}, g_{3}\right]^{t}$ is the magnetic field gradient direction and $\mathbf{T}_{i, j, k}$ are the coefficients of the HOT. To maintain the positive definiteness of higher order tensor, we used the regularization by authors ${ }^{13}$. These HOTs are capable of characterising water diffusion in heterogenous anisotropic regions. 


\section{METHOD}

\subsection{Diagonal component approach}

In this paper, we use the fiber directions from the diagonal component (DC) method ${ }^{14}$. These components can be obtained by flattening higher order tensors of any order. The components of the diagonal part are $3 \times 3$ matrices and their principal eigenvectors can quantify number of underlying fibers.

A 4th order tensor $\mathrm{T}$ can be represented by $9 \times 9$ matrix and it is an algebraic representation that reflects the structure of the 4 th order tensor. The work ${ }^{14}$ provides the mathematical framework of flattening of 4 th order tensor. Thus, a 4th order tensor $T^{(4)}$ can be expressed as a matrix of $(n-2)$ order tensors:

$$
T^{(4)}=\left(\begin{array}{ccc}
T_{x x}^{(2)} & T_{x y}^{(2)} & T_{x z}^{(2)} \\
T_{y x}^{(2)} & T_{y y}^{(2)} & T_{y z}^{(2)} \\
T_{z x}^{(2)} & T_{z y}^{(2)} & T_{z z}^{(2)}
\end{array}\right)
$$

In terms of apparent diffusion coefficients, the first block can be written as:

$$
T_{x x}^{(2)}=\left(\begin{array}{ccc}
D_{x x x x} & D_{x x x y} & D_{x x x z} \\
D_{x x x y} & D_{x x y y} & D_{x x y z} \\
D_{x x x z} & D_{x x y z} & D_{x x z z}
\end{array}\right)
$$

The principal eigenvectors of the three diagonal blocks : $T_{x x}^{(2)}, T_{y y}^{(2)}$ and $T_{z z}^{(2)}$ are used in next section for initial directions in optimisation purpose.

\subsection{Decomposition of 4 th order tensor}

The orientation distribution function (ODF) is obtained using adaptive kernel method ${ }^{13}$. In a related work ${ }^{11}$, decomposition of 4 th order tensors as sum of squares of 2 nd order ones is based on establishing the principal axes from the maxima of ODF and it is used for registration purposes. In this work, we employ such decomposition for tractography and further, the initial directions of maxima are computed using the DC method. In experimental section, we show that the DC approach is better than the Cartesian tensor orientation distribution function $(\mathrm{CTODF})^{15}$ approach to obtain maxima. The initial goal is to decompose the 4th order tensor into a sum of 3 quadratic forms. We ensure that these three forms retain SPD property. This number is restricted to three for a 4 th order tensor because a maximum of three diagonal components can be obtained in such a case. For a 6 th order tensor, these components can yield nine directions. Their principal directions ideally should align with maxima after reorientation.

Each SPD matrix $D$ can be written as, $D=V \Lambda V^{T}$, where $V$ is the orthogonal matrix containing the eigenvectors of the tensor (the orthonormal polar basis for $D$ ) and $\Lambda$ is the positive diagonal matrix with the positive real eigenvalues. For our purposes, we shall work on the principal eigenvector obtained from the DC projections and we shall assume that the other two (smaller) eigenvalues are equal, $\lambda_{i 1} \geq \lambda_{i 2}=\lambda_{i 3}>0$. The principal eigenvector of the diffusion tensor then gives the main fiber direction. As usual, we can rewrite $D$ in its vectorized form as:

$$
\mathbf{D}_{\mathbf{i}}=\operatorname{vec}\left(D_{i}\right)=\left[D_{i x x}, D_{i y y}, D_{i z z}, \sqrt{2} D_{i x y}, \sqrt{2} D_{i x z}, \sqrt{2} D_{i y z}\right]
$$

By the well known theorem going back to David Hilbert, we know that each quartic symmetric positively semidefinite tensor can be written as a sum of squares of three quadratic forms. With our restriction, we consider the best possible approximation in the form ${ }^{11}$ :

$$
\mathbf{T}=\sum_{i=1}^{3} \mathbf{D}_{\mathrm{i}}^{T} \mathbf{D}_{\mathrm{i}}=\sum_{i=1}^{3}\left(\operatorname{vec}\left(V_{i} \Sigma_{i} V_{i}\right)\right)^{T} \operatorname{vec}\left(V_{i} \Sigma_{i} V_{i}^{T}\right) .
$$

The tensor $\mathbf{T}$ is describing the diffusivity $d(\mathbf{g})$ by (4). The considered distance of two such tensors $d\left(\mathbf{T}_{1}, \mathbf{T}_{2}\right)$ is given by ${ }^{11}$

$$
d\left(\mathbf{T}_{1}, \mathbf{T}_{2}\right)^{2}=\frac{1}{4 \pi} \int_{S^{2}}\left(d_{1}(\mathbf{g})-d_{2}(\mathbf{g})\right)^{2} d \mathbf{g}
$$


and the integral of the polynomial expression over the unit sphere is expressed by a simple algebraic formula ${ }^{11}$ (see section 3 in the reference).

Consequently, in order to get the best possible approximation, we have to solve the following non-linear optimization problem:

$$
\min _{\lambda_{i 1}^{\prime}, \lambda_{i 2}^{\prime}} \operatorname{dist}\left(\mathbf{T}, \operatorname{vec}\left(\sum_{i=1}^{3}\left(\mathbf{X}_{\mathrm{i}}^{T} \mathbf{X}_{\mathrm{i}}\right)\right)\right)^{2}
$$

where $\lambda_{i 1}^{\prime}, \lambda_{i 2}^{\prime}$ are the six unknown variables, $\mathbf{X}_{i}=\operatorname{vec}\left(\mathbf{V}_{i} \operatorname{diag}\left(\lambda_{i 1}^{\prime 2}, \lambda_{i 2}^{\prime 2}, \lambda_{i 2}^{\prime 2}\right) \mathbf{V}_{i}^{T}\right)$, and the first columns of $V_{i}$ are the known principal unit eigenvectors, while the other two columns are extending it into an othonormal frame. We obtain the principal unit vectors by the DC method mentioned above.

Thus, the resulting SPD second order tensors have principle eigenvectors aligned with underlying anisotropic directions which are used to trace the crossing fibers.

The following algorithm summarizes our procedure for treatment of voxels including crossing or merging fibers. For the sake of simplicity we present the algorithm and the experiments below for 2-dimensional data and two fibers crossing or merging.

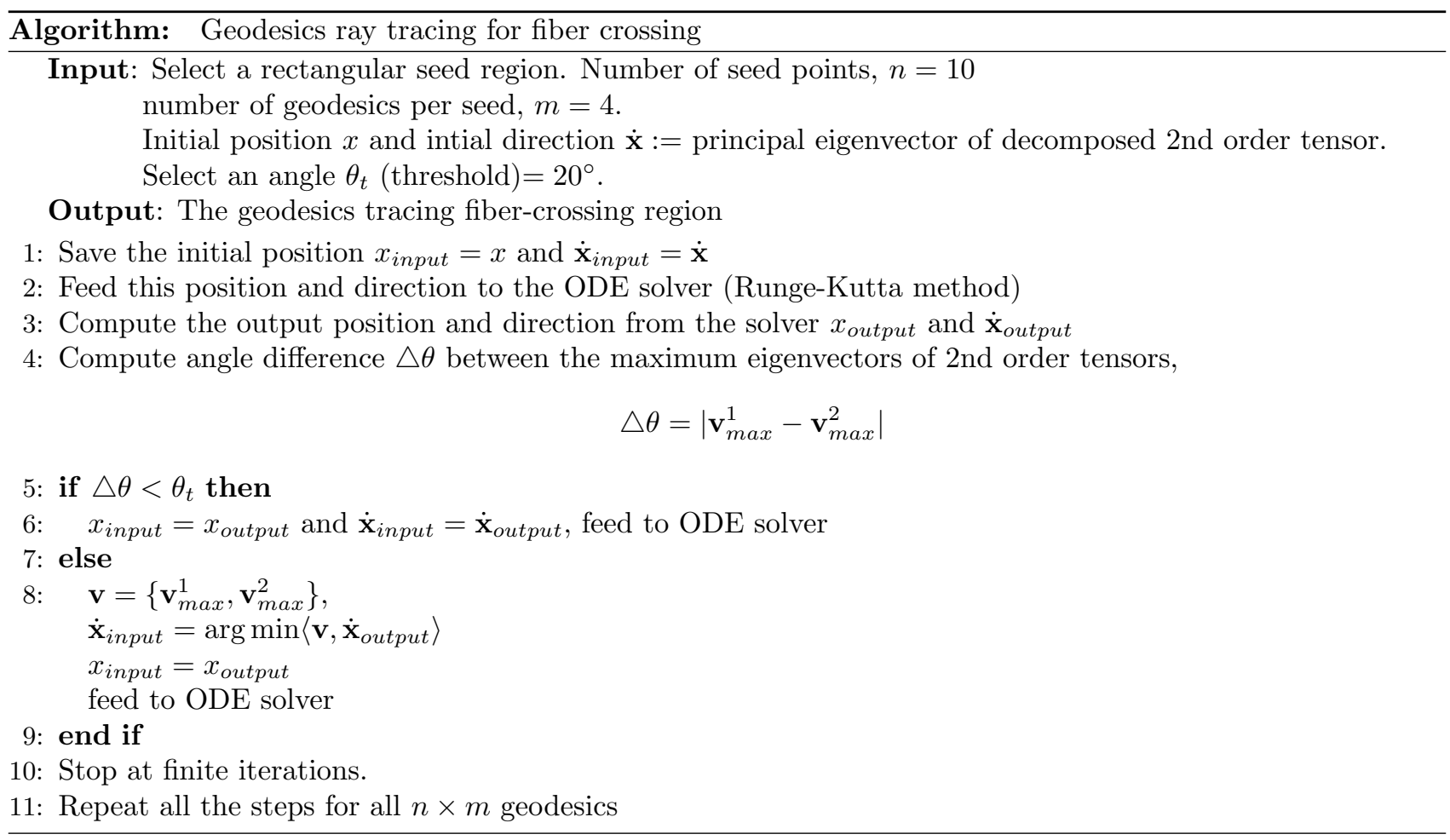

The algorithm shows the steps for modified ray tracing method for tracing in heterogeneous crossing regions. The algorithm can be extended for any number of fibers.

\section{EXPERIMENTS AND RESULTS}

We used the method ${ }^{16}$ to simulate multi-fiber synthetic images with 64 gradient directions, and setting $\mathrm{b}=1500$ $\mathrm{s} / \mathrm{mm} 2$. This approach is based on the generalization of the spherical deconvolution method with the adaptive shape of the kernel. It mimics the water diffusion in compartmental structures of tissues effectively.

The Fig 1 shows the ODFs for 4th order tensors constructed from two fibers crossing at various given angle differences (ground truths: column 1). The corresponding two second order tensors obtained from the 


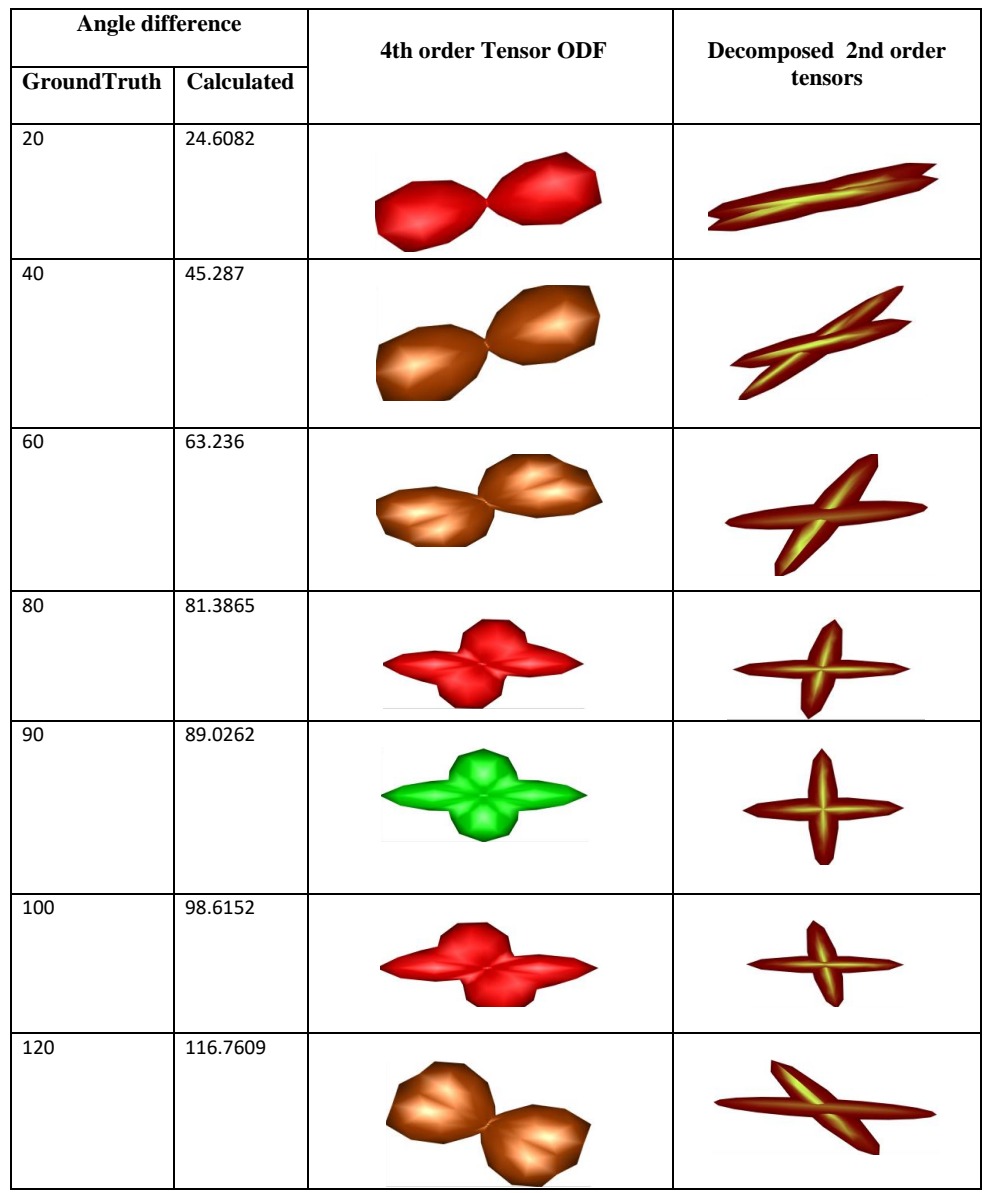

Figure 1: Angle differences, 4th order tensor and the corresponding 2nd order tensors

decomposition are also shown in parallel. The angle differences (column 2) are evaluated between the maximum eigenvectors of these tensors.

At a seed point in a rectangular region, 10 positions are selected and 4 geodesics per position are shot in a cone. In experiments, the traversing geodesics are stopped by fixing the number of iterations, the other way is to stop the geodesics at location where fractional anisotropy scalar values are closer to zero (isotropic background). The coefficients of 2 nd order tensor are obtained from those of 4 th order by linear relation. For more details the reader is referred to ${ }^{2}$. The Fig 2 a) shows two linear crossing fibers at a small angle and Fig $2 \mathrm{c}$ ) the curved crossing fibers.

In intersection regions, due to lack of orientation information when using only the DTI data, the traversing geodesics deflect from actual paths Fig 2 b) and d). The 4th order tensor field reconstructions are shown in Fig 3 a) and c) for linear and curved crossing fibers. The ground truths representing the underlying fiber bundles are shown in red and green.

In this case, the decomposed 2nd order tensors retains the actual path directions and thus allows the geodesics to follow correct diffusion propagation paths. In the crossing regions, the directions of actual paths are visible which otherwise is ambiguous in DTI cases. 


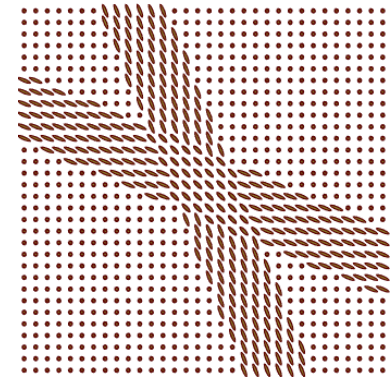

(a)

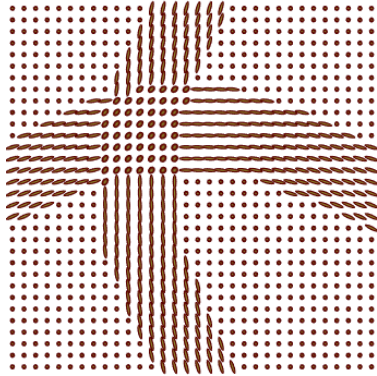

(c)

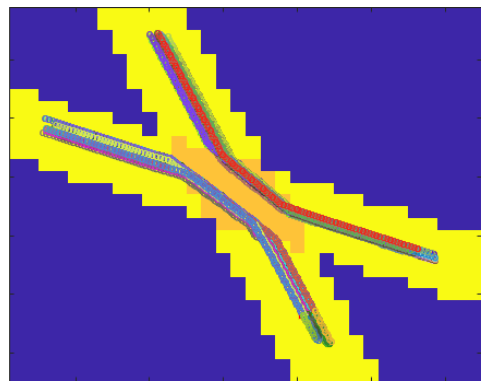

(b)

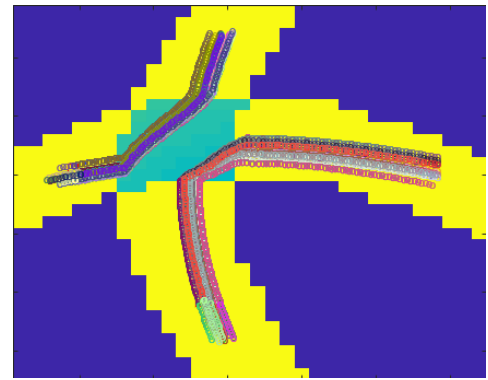

(d)

Figure 2: The 2nd order tensor images (DTI): a) two linear fibers c) curved fibers. The corresponding images b) and d) showing geodesics tracking the incorrect fiber bundle, results are shown on fractional anisotropy scalar image

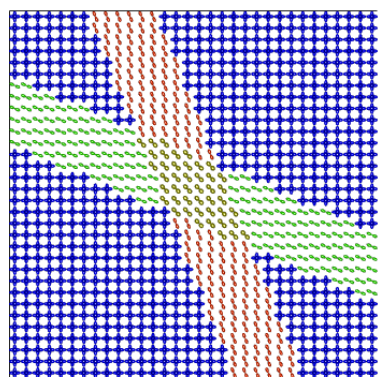

(a)

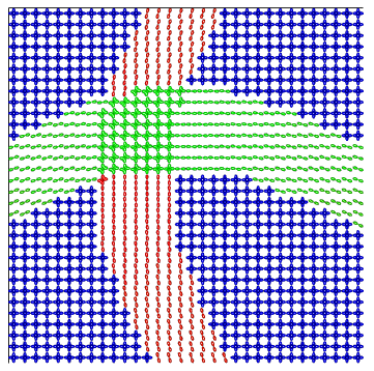

(c)

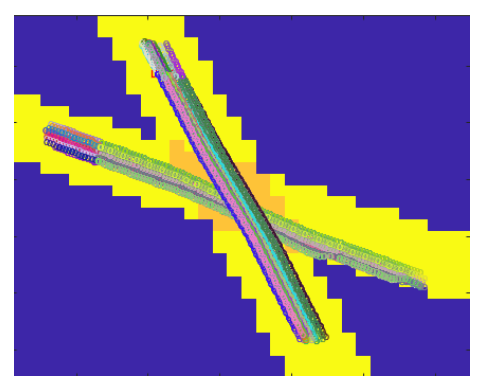

(b)

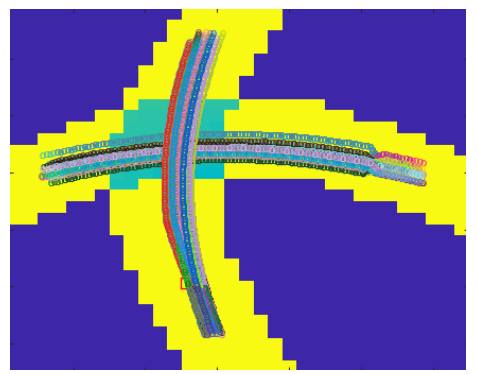

(d)

Figure 3: The 4nd order tensor images: a) two linear fibers c) curved fibers. The corresponding images b) and d) shows geodesics tracking the correct underlying fibers, results are shown on fractional anisotropy scalar image 


\section{CONCLUSIONS}

The diffusion imaging modality is existing for a long time and has found its use in the diagnosis and study of many diseases, e.g. ischemia, alzeihmers, parkinson etc. The theoretical machinery ${ }^{17,18}$ is well established for this data (3x3 SPD matrices). Due to the limitation of DTI in modelling water diffusion in complex tissue structures, the methods developed for various applications, for instance, geodesic ray-tracing for tractography, also need to be customised for higher order models. This work addresses this issue by employing a modified ray-tracing algorithm in the HOTs case to reconstruct fibers in complex structures, exploiting recent results by the authors ${ }^{9,14}$. As a next step, we aim to examine the approach for real images.

\section{ACKNOWLEDGMENTS}

The first and third author have been supported by the university grant MUNI/A/1160/2020 of Masaryk University, Jan Slovák gratefully acknowledges support from the Grant Agency of the Czech Republic, grant Nr. GA20-11473S. We thank Jan Kybic for his valuable suggestion to improve the manuscript. Sumit Kaushik acknowledges the support of the OP VVV funded project CZ.02.1.01/0.0/0.0/16-019/0000765 Research Center for Informatics, Czech Technical University in Prague, Czech Republic.

\section{REFERENCES}

[1] Tuch, D. S., Reese, T. G., Wiegell, M. R., Makris, N., Belliveau, J. W., and Wedeen, V. J., "High angular resolution diffusion imaging reveals intravoxel white matter fiber heterogeneity," Magnetic Resonance in Medicine: An Official Journal of the International Society for Magnetic Resonance in Medicine 48(4), $577-582(2002)$.

[2] Özarslan, E., Vemuri, B. C., and Mareci, T. H., "Generalized scalar measures for diffusion MRI using trace, variance, and entropy," Magnetic Resonance in Medicine: An Official Journal of the International Society for Magnetic Resonance in Medicine 53(4), 866-876 (2005).

[3] Basser, P., Mattiello, J., and Lebihan, D., "Estimation of the effective self-diffusion tensor from the nmr spin echo," Journal of Magnetic Resonance, Series B 103(3), 247-254 (1994).

[4] Sepasian, N., ten Thije Boonkkamp, J., Ter Haar Romeny, B. M., and Vilanova, A., "Multivalued geodesic ray-tracing for computing brain connections using diffusion tensor imaging," SIAM Journal on Imaging Sciences 5(2), 483-504 (2012).

[5] Sepasian, N., Vilanova, A., Florack, L., and Romeny, B. T. H., "A ray tracing method for geodesic based tractography in diffusion tensor images," in [2008 IEEE Computer Society Conference on Computer Vision and Pattern Recognition Workshops], 1-6, IEEE (2008).

[6] Lenglet, C., Deriche, R., and Faugeras, O., "Inferring white matter geometry from diffusion tensor MRI: Application to connectivity mapping," in [European Conference on Computer Vision], 127-140, Springer (2004).

[7] O'Donnell, L., Haker, S., and Westin, C.-F., "New approaches to estimation of white matter connectivity in diffusion tensor MRI: Elliptic pdes and geodesics in a tensor-warped space," in [International Conference on Medical Image Computing and Computer-Assisted Intervention], 459-466, Springer (2002).

[8] Descoteaux, M., Lenglet, C., and Deriche, R., "Diffusion tensor sharpening improves white matter tractography," in [Medical Imaging 2007: Image Processing], 6512, 65121J, International Society for Optics and Photonics (2007).

[9] Bihonegn, T., Kaushik, S., Bansal, A., Vojtíšek, L., and Slovák, J., "Geodesic fiber tracking in white matter using activation function," Computer Methods and Programs in Biomedicine, 106283 (2021).

[10] Bihonegn, T. T., Bansal, A., Slovák, J., and Kaushik, S., "4th order tensors for multi-fiber resolution and segmentation in white matter," in [2020 7th International Conference on Biomedical and Bioinformatics Engineering], ICBBE '20, 36-42, Association for Computing Machinery, New York, NY, USA (2020).

[11] Grigis, A., Renard, F., Noblet, V., Heinrich, C., Heitz, F., and Armspach, J., "A new high order tensor decomposition: Application to reorientation.," in [2011 IEEE International Symposium on Biomedical Imaging: From Nano to Macro], 258-261 (2011). 
[12] Stejskal, E. O. and Tanner, J. E., "Spin diffusion measurements: spin echoes in the presence of a timedependent field gradient," The journal of chemical physics 42(1), 288-292 (1965).

[13] Barmpoutis, A., Hwang, M. S., Howland, D., Forder, J. R., and Vemuri, B. C., "Regularized positive-definite fourth order tensor field estimation from DW-MRI," NeuroImage 45(1), S153-S162 (2009).

[14] Kaushik, S. and Slovák, J., "Hardi segmentation via fourth order tensors and anisotropy preserving similarity measures," Journal of Mathematical Imaging and Vision 2018(5), 12 (2019).

[15] Weldeselassie, Y. T., Barmpoutis, A., and Atkins, M. S., "Symmetric positive-definite cartesian tensor orientation distribution functions (ct-odf)," in [Medical Image Computing and Computer-Assisted Intervention - MICCAI 2010], Jiang, T., Navab, N., Pluim, J. P. W., and Viergever, M. A., eds., 582-589, Springer Berlin Heidelberg, Berlin, Heidelberg (2010).

[16] Barmpoutis, A., Jian, B., and Vemuri, B. C., "Adaptive kernels for multi-fiber reconstruction," in [International Conference on Information Processing in Medical Imaging], 338-349, Springer (2009).

[17] Jost, J., [Riemannian Geometry and Geometric Analysis], Springer Universitat texts, Springer (2005).

[18] Lenglet, C., Prados, E., Pons, J.-P., Deriche, R., and Faugeras, O., "Brain connectivity mapping using riemannian geometry, control theory, and pdes," SIAM J. Img. Sci. 2, 285-322 (Apr. 2009). 